\title{
PARTICLE ACCELERATION IN SOLAR FLARES
}

\author{
P. A. STUR ROCK \\ Institute for Plasma Research, Stanford University, Stanford, Calif., U.S.A.
}

\begin{abstract}
A review of observational data supports the proposal that there are two distinct phases of particle acceleration in solar flares. 'Phase 1' is associated with the flash phase and is here interpreted as acceleration during field-line reconnection. 'Phase 2' is associated with type II and type IV radio bursts, and is ascribed to stochastic acceleration in the turbulent plasma behind a magnetohydrodynamic shock formed ahead of an ejected plasmoid.
\end{abstract}

\section{Introduction}

It is generally agreed that, in the preflare state, energy is stored in magnetic fields. More specifically, the energy released during a flare is derived from the 'free energy' associated with currents in the solar atmosphere in an active region. It is widely believed that the mechanism by which this energy is converted into other forms is a plasma instability. If the current in the preflare state is in the form of a 'current sheet', the appropriate instability is of the 'tearing mode' type analyzed by Furth $e$ al. (1963). After the instability has reached its nonlinear stage, the reconnection probably proceeds substantially as described by Petschek (1964). One of the simplest geometries for the preflare magnetic field is that proposed by Carmichael (1964) and independently by Sturrock (1966) (Figure 1).

Magnetic energy released during the tearing process must somehow be transferred to the plasma. Dungey (1958) realized that, when reconnection occurs, a DC electric field will be developed in the reconnection region which could lead to particle acceleration. Friedman and Hamburger (1969) and Coppi and Friedland (1971) have proposed that micro-instabilities, of the two-stream type, are likely to occur during reconnection since intense currents will flow in small regions. These micro-instabilities will lead to high-frequency electric fields which can lead to particle acceleration. Within the context of this model, the most obvious source for particle acceleration is, therefore, the reconnection process itself.

When we come to consider observational data, we find that the question of acceleration cannot be answered so simply. There have been many reviews of the properties of solar flares, perhaps the most recent being that of Švestka (1973). Particle emission from solar flares has recently been reviewed by Lin (1974) and Simnett (1974). These recent reviews support a proposal made by de Jager (1969), that there are two stages of acceleration of particles in solar flares, which we shall refer to as 'Phase 1 Acceleration' and 'Phase 2 Acceleration'.

Phase 1 acceleration occurs during the flash phase of a flare and is of limited duration, of order 1-2 min. Spacecraft observations indicate that large numbers of electrons are accelerated to energies in the range 10-100 keV during this phase. During this phase, type III radio bursts occur, which are usually ascribed to the passage of discrete beams of electrons, with energies in the above range, through 
the corona and into interplanetary space. It is a common view that these particles travel through coronal streamers, possibly in the current sheets which are believed to lie within streamers. It is known that impulsive nonthermal X-ray bursts occur during this stage of a flare. These bursts may be understood as the result of electron

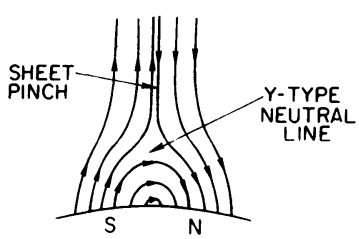

(a)

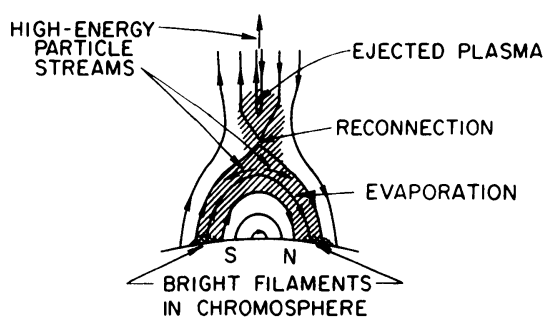

(b)
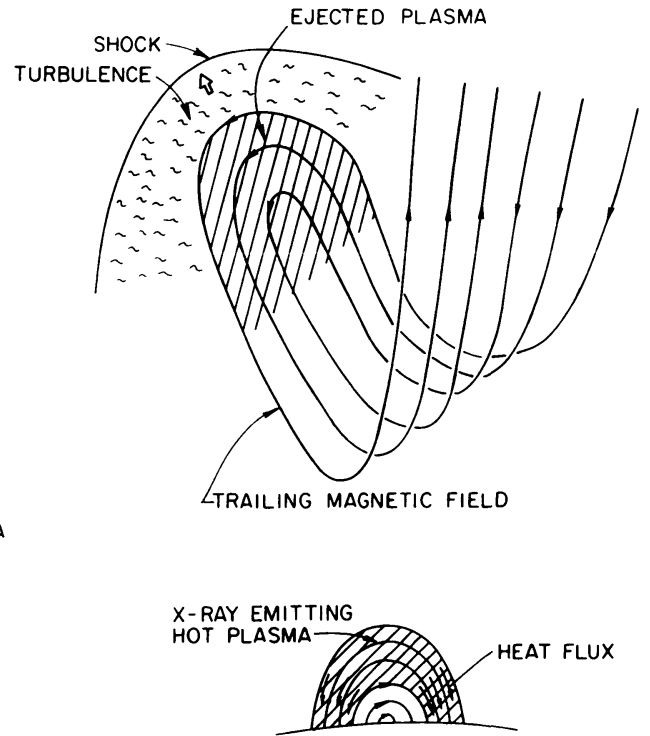

(c)

Fig. 1. (a) Example of 'open' current sheet configuration above a bipolar magnetic region. (b) Reconnection results in particle acceleration which in turn leads to evaporation. (c) Following reconnection, magnetic field near Sun's surface is in current-free state and a mass of plasma, trailing magnetic field lines, is ejected from the corona.

streams, similar to those which escape into interplanetary space, streaming down into the chromosphere. Petrosian (1973) has shown that the polar diagram of bremsstrahlung radiation becomes peaked in the 'forward' direction (pointing into the photosphere) as one considers photons of energy of order $100 \mathrm{keV}$ produced by mildly relativistic electrons. This means that impulsive $\mathrm{X}$-rays are not a good indicator of the electron spectrum above about $100 \mathrm{keV}$. On the other hand, spacecraft observations indicate that there is typically a cut-off in the electron spectrum between $100 \mathrm{keV}$ and $500 \mathrm{keV}$. There is no indication, from observations made with particle detectors, that Phase 1 acceleration typically leads to a flux of protons or other ions. If such a flux is produced, the number of accelerated ions is much smaller than the number of accelerated electrons. It may be noted that Frost (1972) has recently introduced the term 'type III X-ray burst' to characterize the impulsive X-ray bursts occurring during this stage, to discriminate them from X-ray bursts sometimes observed later in the flare, as will be described below. 
Phase 2 acceleration occurs in only a small fraction of flares, namely those flares which exhibit 'high-energy' phenomena. The most direct evidence for Phase 2 acceleration is the detection, by spacecraft, of streams of relativistic electrons and ions. These measurements show that Phase 2 acceleration typically occurs 5-10 min after the flash phase of the flare. Proton streams have power-law spectra with detectable fluxes at $100 \mathrm{MeV}$, and sometimes at $1 \mathrm{BeV}$ or more. Electron fluxes are much smaller than the proton fluxes, but the energies may extend up to $10 \mathrm{MeV}$ or more. The abundance distribution of the ion stream varies from flare to flare, and has a higher contribution of high- $Z$ elements than is characteristic of the photosphere. Particle fluxes from Phase 2 acceleration seem sometimes to arrive promptly and sometimes to propagate primarily through diffusion.

The occurrence of high-energy particle streams, which we attribute to Phase 2 acceleration, correlates well with type II and type IV radio bursts (Kundu, 1965). Type II bursts are generally attributed to shock fronts moving through the corona; this may possibly be a 'blast wave', but it is more likely to be a 'bow shock' ahead of a moving plasmoid, as shown in Figure 1 (c). Type IV radiation is generally attributed to gyro-synchrotron radiation by mildly relativistic electrons either in magnetic field patterns anchored to the photosphere, or in the closed magnetic field of a moving plasmoid. The ejected plasma cloud shown in Figure 1 (c) has only open field lines, but reconnection at the neutral sheet could produce a plasmoid with a closed, toroidal field pattern, as shown in Figure 2. Such a field pattern, in a moving plasmoid, would provide a good fit to the radio source observed on March 1, 1969 (Riddle, 1970).

Impulsive X-ray bursts occur also during the time-frame of Phase 2 acceleration.

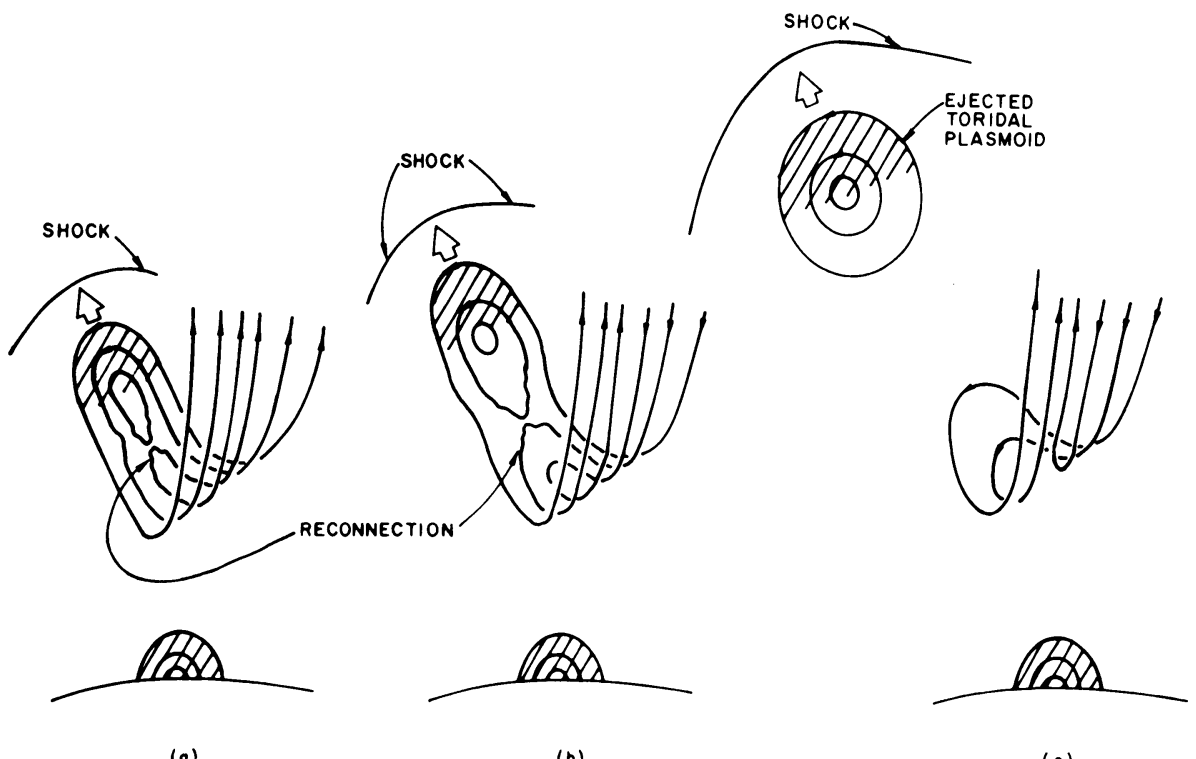

(o)

(b)

(c)

Fig. 2. (a, b) Reconnection can occur in current sheet which develops in ejected plasmoid. (c) Such reconnection could lead to an ejected plasmoid with a closed magnetic-field configuration, possibly toroidal. 
Since they occur at about the same time as type IV radio bursts, Frost (1972) has introduced the term 'type IV X-ray bursts' to describe them. These X-ray bursts differ from type III X-ray bursts in that they do not show a cut-off in the X-ray spectrum (indicative of a cut-off in the electron spectrum).

\section{Phase 1 Acceleration}

We next discuss briefly possible mechanisms of Phase 1 acceleration. Since the reconnection process converts magnetic energy into plasma kinetic energy, this must give rise to some increase in particle energy. A simple estimate of the energy $E(\mathrm{eV})$ acquired by electrons and protons is given by the energy balance equation

$$
\frac{1}{8 \pi} B^{2}=2 n \times 10^{-11.8} E,
$$

where $n$ is the electron density. This becomes

$$
E=10^{10.1} B^{2} n^{-1} \text {. }
$$

Adopting a typical value $B=10^{1.5}$ (gauss) for the coronal magnetic field of an active region, and $n=10^{8}\left(\mathrm{~cm}^{-3}\right)$ for the preflare coronal density, Equation (2) leads to the estimate $E=10^{5.1}$, in good agreement with the fact that the electron energy spectrum typically cuts off at about $100 \mathrm{keV}$. Note that, as the flare develops, the density of coronal plasma apparently increases due to evaporation from the chromosphere. This increase in $n$ will lead to a corresponding decrease in $E$. Data concerning soft $\mathrm{X}$-ray emission from flares indicate that, during the late stage of a flare, the coronal density may be in the range $n=10^{10}$. This would lead to $E=10^{3}$. The mean free path of particles of this energy in a plasma of this density is only $10^{8} \mathrm{~cm}$. Hence these particles would not escape freely to the chromosphere and into interplanetary space: rather, they would lose energy simply by heat conduction.

Acceleration of electrons during the Phase 1 stage is most probably due to the interaction of electrons with RF electric fields developed in the tearing region as the result of a two-stream electron-ion instability. Since the phase velocity of the excited waves are comparable with the electron streaming velocities, there will be little interaction between these waves and ions. These considerations are consistent with the observational fact that Phase 1 acceleration is mainly electron acceleration, with comparatively little proton acceleration. We also note that energy considerations are consistent with the fact that there is typically a sharp change in the electron energy spectrum at an energy of order $100 \mathrm{keV}$. A more extensive discussion of particle acceleration during the flash phase of solar flares is given by Smith (1974).

\section{Phase 2 Acceleration}

We next discuss possible mechanisms for Phase 2 acceleration. Observational evidence indicates that this probably occurs in association with a shock front. It is known, 
from observations of the Earth's bow shock, that a collision-free shock leads to shocked gas which is in a state of MHD turbulence. This leads to the possibility of stochastic particle acceleration (Hall and Sturrock, 1967; Newman, 1973), which comprises a class of acceleration mechanisms of which Fermi's is a particular case.

Suppose that the mean magnetic field strength in the shocked gas is $B$ (Gauss), the mean plasma density $n\left(\mathrm{~cm}^{-3}\right)$, and the thickness of the shocked region is $L(\mathrm{~cm})$. Suppose that the 'depth of modulation' of the turbulence is $\delta$, so that the magnitude of the fluctuating magnetic field is $\delta B$. The phase velocity of waves comprising the turbulence will be comparable with the Alfvén velocity $v_{\mathrm{A}}$, given by

$$
v_{\mathrm{A}}=10^{11.3} \mathrm{Bn}^{-1 / 2} \text {. }
$$

The frequency spectrum of the MHD turbulence will extend from low frequencies up to a maximum of approximately $\omega_{g p}$, the radian gyro-frequency of protons, given by

$$
\omega_{g p}=10^{4.0} B \text {. }
$$

This means that the turbulence loses correlation for distances larger than $\lambda$ given by

$$
\lambda=\frac{v_{\mathrm{A}}}{\omega_{g p}}=10^{7.3} n^{-1 / 2} .
$$

If a particle has sufficiently high energy, it will traverse the turbulent plasma sampling the electric field in different cells, the cell size being given by Equation (5). The magnitude of the electric field $\mathscr{E}$ (esu) is given by

$$
\mathscr{E}=\frac{v_{\mathrm{A}}}{c} \delta B=10^{0.8} \delta B^{2} n^{-1 / 2} .
$$

If a particle travels a total distance $L$, it samples $(L / \lambda)$ different cells, and gains or loses an energy $e \mathscr{E} \lambda(\mathrm{erg})$ per cell. Treating this as a random walk process, the expectation value of the final energy $E_{\mathrm{av}}$ is given by

$$
E_{\mathrm{av}}=10^{11.8}\left(\frac{L}{\lambda}\right)^{1 / 2} e E \lambda
$$

On using Equations (5) and (6), we find that this may be re-expressed as

$$
E_{\mathrm{av}}=10^{7.0} \delta L^{1 / 2} B^{2} n^{-3 / 4} \text {. }
$$

We may also estimate the absolute maximum energy which could be acquired by any particle by assuming that the electric field has the same sign over the total path length $L$. One then finds, using Equation (6), that the maximum energy is given by

$$
E_{\max }=10^{3.3} \delta L B^{2} n^{-1 / 2} \text {. }
$$

Let us consider the above formulas for a large flare characterized by $L=10^{10}$, $B=10^{1.5}$. If the density in the shocked gas is that of the preflare coronal density in the active region, of order $n=10^{8}$, we find, adopting $\delta=10^{-1}$, that $E_{\mathrm{av}}=10^{8}$ and $E_{\max }=10^{11.3}$. On the other hand, if the density is that characteristic of the post-flare 
plasma, calculations presented elsewhere (Sturrock, 1973) would indicate that $n=10^{9.4}$. We then find that $E_{\mathrm{av}}=10^{7}$ and $E_{\max }=10^{10.6}$.

Stochastic acceleration typically leads to a power-law energy spectrum. The above considerations indicate that there should be a change in slope of the energy spectrum in the range 10-100 MeV, for a large flare. Data reported by Price (1973) show evidence for such a change of slope. We also see that there is no difficulty in understanding that some particles can acquire energies as high as $10 \mathrm{BeV}$.

We may also note that particles will experience stochastic acceleration, as described above, only when they have gyro-radii $r_{g}$ comparable with or larger than the turbulence cell size, i.e.

$$
r_{g} \geqslant \lambda \text {. }
$$

Electrons will typically be relativistic, for which

$$
r_{g}=10^{-2.5} E B^{-1} \text {, }
$$

so that the 'injection energy' of electrons, $E_{e, \text { inj }}$, is given by

$$
E_{e, \text { inj }}=10^{9.8} \mathrm{Bn}^{-1 / 2} \text {. }
$$

For the two cases considered above, this energy is estimated to be $10^{7.3} \mathrm{eV}$ or $10^{6.6} \mathrm{eV}$. Ions will typically be nonrelativistic at injection, so that $r_{g}$ is given by

$$
r_{g}=10^{2.2} A^{1 / 2} Z^{-1} E^{1 / 2} B^{-1}
$$

Equation (10) therefore leads to the following formula for the injection energy of ions:

$$
E_{i, \text { inj }}=10^{10.2} Z^{2} A^{-1} B^{2} n^{-1} \text {. }
$$

The injection energy for protons, for the above two cases, is found to be $10^{5.2} \mathrm{eV}$ and $10^{3.8} \mathrm{eV}$. Since the injection energy for ions is considerably lower than that for electrons, one can understand that Phase 2 acceleration typically produces larger fluxes of ions than of electrons.

In Table I, certain acceleration parameters are presented for three sets of primary

TABLE I

\begin{tabular}{llll}
\hline & $L=10^{8.5}, B=10^{3}$ & $L=10^{8.5}, B=10^{2}$ & $L=10^{10}, B=10^{2}$ \\
\hline$W(\mathrm{erg})$ & $10^{29.8}$ & $10^{27.8}$ & $10^{32.3}$ \\
$n_{\mathrm{PF}}\left(\mathrm{cm}^{-3}\right)$ & $10^{11.6}$ & $10^{10.2}$ & $10^{9.7}$ \\
Phase 1 & & & \\
$E_{e(\text { knee })}(\mathrm{eV})$ & $10^{4.5}$ & $10^{3.9}$ & $10^{4.4}$ \\
Phase 2 & & & \\
$E_{p(\text { knee })}(\mathrm{eV})$ & $10^{7.6}$ & $10^{6.6}$ & $10^{7.7}$ \\
$E_{p(\max )}(\mathrm{eV})$ & $10^{11.0}$ & $10^{9.7}$ & $10^{11.5}$ \\
$E_{e(\text { inj })}(\mathrm{eV})$ & $10^{7.0}$ & $10^{6.7}$ & $10^{7.0}$ \\
$E_{p(\text { inj })}(\mathrm{eV})$ & $10^{4.6}$ & $10^{4.0}$ & $10^{4.5}$ \\
\hline
\end{tabular}


flare parameters $B$ and $L$. The total flare energy $W(\mathrm{erg})$ and the post-flare density are given, estimated on the model recently proposed (Sturrock, 1973). Using these values of $B, L$ and $n$, the preceding formulas are used to calculate the energy at which the energy spectra should show a 'break' or 'knee' (for both Phase 1 and Phase 2 acceleration), the maximum energy to which protons might be accelerated, and the injection energy for electrons and protons (for Phase 2 acceleration).

Finally, we may note that the model proposed for Phase 2 acceleration meets the principle requirements set down by Cartwright and Mogro-Campero (1972), as a result of analysis of abundance data. The final acceleration is stochastic; the injection condition depends primarily on rigidity; and accelerated particles may be in a fairly dense plasma (the post-flare plasma) before injection.

A more detailed treatment of Phase 2 acceleration, based on the theory of stochastic acceleration in a turbulent plasma (Hall and Sturrock, 1967; Newman, 1973) will be presented at a later date.

\section{Acknowledgements}

This work was supported by the National Aeronautics and Space Administration under Grant NGL 05-020-272 and the Office of Naval Research under Contract N0001467-A-0112-0062.

\section{References}

Carmichael, H.: 1964, in W. Hess (ed.), AAS-NASA Symp. on the Physics of Solar Flares, NASA SP-50, p. 459.

Cartwright, B. G. and Mogro-Campero, A.: 1972, Astrophys. J. Letters 177, L43.

Coppi, B. and Friedland, A. B. : 1971, Astrophys. J. 169, 379.

De Jager, C.: 1969, in C. de Jager and Z. Śvestka (eds.), 'Solar Flares and Space Research', COSPAR Symp., 1.

Dungey, J. W.: 1958, Cosmic Electrodynamics, Cambridge University Press, p. 98.

Friedman, M. and Hamburger, S. M.: 1969, Solar Phys. 8, 104.

Frost, K. J.: 1972, private communication.

Furth, H. P., Killeen, J., and Rosenbluth, M. N.: 1963, Phys. Fluids 6, 459.

Hall, D. E. and Sturrock, P. A.: 1967, Phys. Fluids 10, 2620.

Kundu, M. R.: 1965, Solar Radio Astronomy, Interscience, New York.

Lin, R. P.: 1974, Space Sci. Rev. 16, 189.

Newman, C. E.: 1973, J. Math. Phys. 14, 502.

Petrosian, V.: 1973, Astrophys. J. 186, 291.

Petschek, H. E.: 1964, in W. Hess (ed.), AAS-NASA Symp. on Physics of Solar Flares, NASA SP-59, p. 425.

Price, P. B.: 1973, in R. Ramaty and R. G. Stone (eds.), Proc. Symposium on High Energy Phenomena on the Sun, NASA Goddard Space Flight Center, p. 377.

Riddle, A. C.: 1970, Solar Phys. 13, 448.

Simnett, G. M.: 1974, Space Sci. Rev. 16, 257.

Smith, D. F.: 1974, this volume, p. 253.

Sturrock, P. A.: 1966, Nature 211, 695.

Sturrock, P. A.: 1973, in R. Ramaty and R. G. Stone (eds.), Proc. Symposium on High Energy Phenomena on the Sun, NASA Goddard Space Flight Center, p. 3.

Švestka, Z.: 1973, Conf. on Solar Terrestrial Relations, Calgary, Canada, p. 23. 


\section{DISCUSSION}

Uchida: What is the amount of mass involved in the process (tongue shaped mass ejection in Carmichael's model) you are talking about? That can only bring out the mass initially lying in the corona and must be small.

Sturrock: The numbers are given in my Goddard talk, but the mass ejected from the corona is evaporated from the chromosphere in the course of flare.

Uchida: Isn't it true that the closure of the field pattern in your slide by reconnection occurs before the evaporation process takes place?

Sturrock: Reconnection is not restricted to the flash phase; it lasts as long as the flare itself, i.e. for hours.

Kane: You mentioned that in Phase 2 the acceleration occurs in a high density region. However the hard X-ray observations of behind-the-limb flares indicate that the energetic electrons (in Phase 2) are located in a low density region. How do these electrons get from the acceleration region to the hard X-ray source? How much energy is lost in the transportation process?

Sturrock: Acceleration occurs in a current sheet, then the accelerated particles cause evaporation. However, the model has been not worked out in sufficient detail to answer your question.

Dryer: Your point about the term 'bow shock' with its analogy to the Earth's bow shock is appropriate. Some clarification is necessary, however. If your magnetic piston maintains its geometrical size as it moves from sub- to superalfvénic velocities, the shock will develop, at first, far in front of the barrier, quickly approaching its classical position (steady-state) given as a function of the density compression ratio across the shock (i.e. as a function of specific heat ratio and Mach number). As the blob (still the same size) changes its Mach number, the shock's position changes accordingly just as in the case of the Earth. The comments about turbulence between the shock and the driver are not appropriate with the proviso that the undisturbed magnetic field should - if the Earth's bow shock's example may be used - be highly oblique or perpendicular to the shock normal. If the field is more or less parallel to the shock normal, there should indeed be such turbulence as seen behind the Earth's shock. If, however, the blob expands, the shock adjusts by moving away from the driver piston. Its velocity will respond in accordance with the acceleration, constant velocity, or deceleration of the piston.

Sturrock: I agree.

Lin: Your acceleration mechanism takes place in the turbulent region behind the shock. Observations of the Earth's bow shock and interplanetary shocks show that the particles are accelerated at the shock or in front of the shock. Is there anything wrong with a simple Fermi mechanism between the shock and magnetic structures ahead of it for the second phase acceleration? Protons would naturally get more energy than electrons for a Fermi mechanism since the proton velocities are lower for the same energy.

Sturrock: I would need some numbers to make any comment.

Vrabec: Your figures of magnetic field configurations associated with flares showed essentially only their two-dimensional geometry. Would you please comment on the importance of the three-dimensional configuration of the magnetic field with respect to stability and containment.

Sturrock: I assumed the extension in the third dimension to be comparable with the other two.

Meyer: My comment refers to the stochastic particle acceleration process. You mentioned that one can compute a minimum injection energy for acceleration to occur from the requirement that the gyro radii initially should not be smaller than the smallest turbulent scale. It seems that this would be required for those induced electric fields that are perpendicular to the magnetic field. However under very turbulent conditions one might expect also a rapid shearing of magnetic fields which induce electric fields along the magnetic field. Such electric fields would also accelerate particles whose gyro radii are smaller than the turbulent scale, so that the minimum injection energy requirement would be lifted. Of course such electric fields would differentiate strongly between electrons and protons. The former, due to their mobility, will pick up most of the energy available from such fields and might thereby shortcircuit such fields before protons are able to make use of them.

Smith: A very important part of this model is boiling off part of the chromosphere. The only person who has done detailed calculations regarding this possibility is Brown. I would like to know whether he considers this possible.

Brown: On the calculations of mine that Dean Smith mentions, I find chromospheric evaporation by electron streams very favourable in respect of ejecting the necessary $10^{16} \mathrm{gms}$. However, if you are really suggesting using this upflow to feed the current sheet with material, I wouldn't believe it because the dynamical upflow time is around a minute - much larger than the time scale for rapid variation in the sheet as inferred from hard X-ray time profiles and radio data. 
Sturrock: There does seem to be evidence that evaporation occurs (e.g. Hudson and Ohki) but how effective it is may be governed by thermal conduction across the magnetic field.

Leblanc: In your model of magnetic field, what is the height of the top of the magnetic arch where the acceleration of particles occurs?

Sturrock: The height is inferred from observation and built into the model.

Uchida: I have a rather exotic model in which I seek the mass and energy source loss in the photosphere or low chromosphere. People have sought the flare origin in the corona or the high chromosphere mainly because no disturbed photosphere was observed lying beneath the $\mathrm{H} \alpha$ flare area. However, recent observation with high time and spatial resolution of low lying layers as performed by Zirin and Tanaka in $\lambda 3835$ has revealed the existence of perturbed photosphere, though pretty small in size. We are tempted to explain the entire flare as due to a dynamical process if we remember that the main part of the total energy of large flares, $10^{32} \mathrm{erg}$, is mainly due to the interplanetary flux with particle number of $10^{40}$ to $10^{41}$ but this is readily realised if a tiny cube $\left(10^{8} \mathrm{~cm}^{3}\right)$ of the photospheric or low chromospheric material is accelerated to a final velocity of the order of $500 \mathrm{~km} \mathrm{~s}^{-1}$, for example by the melon seed mechanism in a highly stressed field near neutral lines in the photosphere, where photospheric or low chromospheric brightening of such a size was reported by Zirin and Tanaka. It may be added that the location of these is also the location of the kernels in $\mathrm{H} \alpha$ which have very conspicuous characteristics incomparable to the rest of the $\mathrm{H} \alpha$ area according to Zirin.

Sturrock: Without seeing your model I cannot comment.

Kundu: I'd like to ask Dr Sturrock whether he considers the decameter continuum which lasts for several days as enough evidence for trapping of particles near the Sun.

Smerd: The late stationary type IV burst at metre wavelengths, the storm continuum, is compatible with the arrival and passage over several hours of a 'slow' disturbance $\left(\sim 100 \mathrm{~km} \mathrm{~s}^{-1}\right)$ at the coronal plasma levels. The disturbance may well be the 'driver gas' deduced from interplanetary shock wave observations.

If the storm continuum becomes bursty and continues (for $\sim$ days) as a I-III storm, this can, in my opinion, only be interpreted as continued, intermittent acceleration of $\sim 10$ to $\sim 100 \mathrm{keV}$ electrons (and perhaps also sub-relativistic protons). The radio storm evidence suggests that the acceleration of the type I-radiating electrons is due to mhd-turbulence in a closed, strong-field loop, that of the storm IIIradiating electrons due to mhd-triggered re-connections at the cusp or the neutral sheet above the closedloop structure. In my opinion neither the storm continuum nor a radio storm are evidence for the trapping for hours or days respectively.

Enome: From strong proton flares electrons with energies of tens of $\mathrm{MeV}$ are often observed. The spectrum of these electrons is continued smoothly to the non-thermal electron spectrum, which strongly suggests the same origin for both electrons. If a hard X-ray second component is supposed to be accelerated in the plasmoid, strong synchrotron radiation will be expected from the relativistic electrons in the magnetic field of order of $5 \mathrm{G}$. This is not observed.

Kai: We have recorded a complex burst (1973 June 26) with the 80 and $160 \mathrm{MHz}$ radioheliograph which could clarify a rather confusing concept on flare-continuum, moving type IV and storm continuum. The fare-continuum appeared in a very extended source which moved outward slowly $\left(\sim 400 \mathrm{~km} \mathrm{~s}^{-1}\right)$. It is noted that the $160 \mathrm{MHz}$ source faded out more quickly. Later a polarized moving IV source appeared just on the top of the flare-continuum source and continued to move outwards, whereas the flare-continuum source shrank toward the disk, and showed a bipolar structure. This source remained active for a few hours and it was identified with a storm-continuum.

Smerd: Smerd and Dulk (IAU Symp. 43, 1970) suggested that a flare continuum may be evidence for plasma radiation from sub-relativistic electrons injected into closed-field loops during the flash phase. The duration of the flare continuum $(\sim 10 \mathrm{~min})$ is taken as evidence for further acceleration of the injected electrons, probably due to the interaction of the loop with a shock wave.

Note added after discussion. The difference between this and the same process for the second-phase acceleration of the microwave IV-radiating electrons (and protons) is probably that the flare-continuum electrons are in a 'high', active-region spanning loop, those of the microwave IV in 'low' loops near the flare explosion.

Rosenberg: One should be careful to attribute everything to the flare energy. Due to the flux, the magnetic configuration in the corona can change considerably, leading to different heating conditions (e.g. difference between closed and open field lines). This helps to understand also the type I continua, which are not at all flare related, but more related to active regions, strong fields, specific magnetic structures. Changes in heating, or excess heating via Alfvénic or magnetosonic flux also creates turbulence in the coronal structures, which in turn can lead to fast particles. The latter are in that case not directly connected to the flare. 